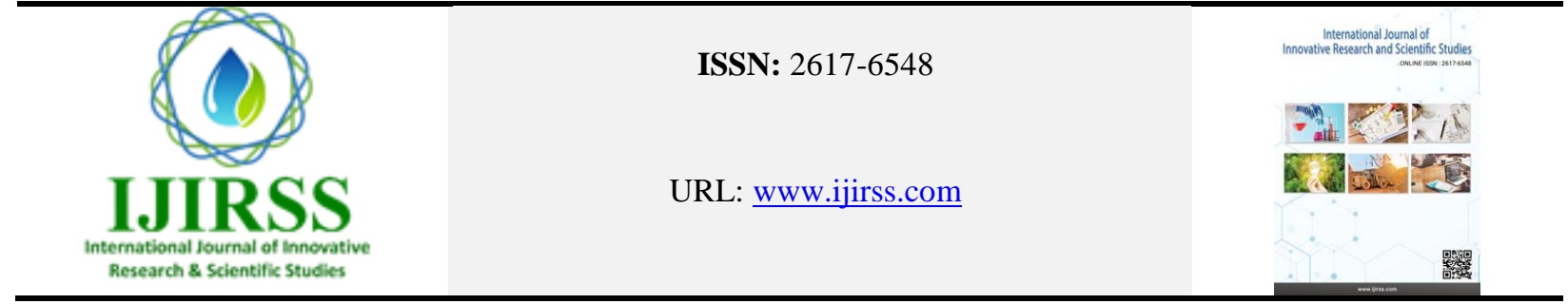

\title{
The Effect of Different Treatments on Seed Dormancy Breaking and Germination Inducing in Louisiana Variety of Okra (Abelmoschus esculentus L.)
}

\author{
Ali Jawed Safdary ${ }^{1}$, Ahmad Jawid Ahamdi ${ }^{2}$, Nasratullah Habibi ${ }^{3 *}$, Zabiullah Rahmani ${ }^{1}$, Saifullah Rasooli ${ }^{2}$ \\ ${ }^{1}$ Department of Horticulture, Faculty of Agriculture, Samangan Higher Education Institute, Samangan, Afghanistan \\ ${ }^{2}$ Department of Agronomy, Faculty of Agriculture, Samangan Higher Education Institute, Samangan, Afghanistan \\ ${ }^{3}$ Department of Agronomy, Faculty of Agriculture, Balkh University, Balkh, Afghanistan \\ *Corresponding author: Nasratullah Habibi (nasratullah.habibi14@gmail.com)
}

\begin{abstract}
To investigate the methods of dormancy breaking in okra seeds; a study was conducted in the winter season of 2019 under laboratory conditions in Samangan Higher Education Institute. The experiment was carried out based on a completely randomized design within 10 treatments and 3 replications. The treatments included gibberellic acid at 4 levels (100, 500, 700 , and $1000 \mathrm{ppm})$, hot water $\left(70^{\circ} \mathrm{C}\right)$, hydrochloric acid $(75 \%)$ at three levels $(5,10$, and 15 minutes), 7 -day chilling at $3{ }^{\circ} \mathrm{C}$ and control treatment (distilled water). The results showed that the highest percentage (100\%) with a germination rate of 86 seeds were available due to the application of 7-day cold treatment. Moreover, the treatments such as 7-day chilling, gibberellic acid $500 \mathrm{ppm}$, and hydrochloric acid 10 and 15 minutes had significant effects on the fresh and dry weight of roots and shoots as well as on their length. we observed that the 7-day cooling at $3{ }^{\circ} \mathrm{C}$ is the best and most appropriate solution to break down the seed dormancy and stimulate seed germination of the above-mentioned crop.
\end{abstract}

Keywords: Dormancy, Germination, Okra.

DOI: 10.53894 /ijirss.v3i4.45

Funding: This study received no specific financial support.

History: Received: 3 August 2020/Revised: 5 October 2020/Accepted: 27 October 2020/Published: 17 November 2020

Licensed: This work is licensed under a Creative Commons Attribution 4.0 License $($ (c) $)$ EY

Acknowledgement: Authors are grateful to the Director of the Samangan Institute of Higher Education for the assistance and equipping of the laboratories that enabled this research to be carried out.

Competing Interests: The authors declare that they have no conflict of interests.

Transparency: The authors confirm that the manuscript is an honest, accurate, and transparent account of the study was reported; that no vital features of the study have been omitted; and that any discrepancies from the study as planned have been explained.

Ethical: This study follows all ethical practices during writing.

\section{Introduction}

Okra (Abelmoschus esculentus L.) belongs to the genus Abelmoschus of the Malvaceae family and is native to the Asian regions. It is one of the most important crops in tropical and subtropical regions. Okra is an annual herbaceous plant with cross-pollination that is 1 to 2 meters tall and has purple-green stems [1]. As the germination stage in plants plays an important role in proper plant establishment and terminal yield, it is necessary to absorb a certain amount of water to promote seed development and metabolic activities. The amount of water absorbed by the seed depends on the chemical composition and permeability of the seed coat. Seed germination is a complex physiological process that responds to many environmental signals including temperature, water potential, light, nitrate and smoke [2]. 
Seed dormancy and germination are also regulated by growth and environmental conditions [3]. The most common type of dormancy is exogenous dormancy, which occurs when the seed coat becomes pale and impermeable to water. It is usually difficult to identify and can be found in many species of the families Fabaceae and Malvaceae [4]. Seed dormancy is a factor imposed by the embryo or other inhibitory components inside the seed while exogenous inhibitors are attributed to the seed coat. The endosperm is the only component that coats seeds that causes exogenous dormancy [5]. Poor and delayed germination due to seed dormancy in okra is one of the major challenges for crop cultivation [6]. The hardness of okra seeds complicates the management of this plant and affects seed germination [7]. Many researchers have been conducted to break dormancy and improve the germination of okra seeds [8,9]. Peto [10] reported that dilute solutions of hydrochloric acid and sulfuric acid were involved in improving the germination of sugar beet seeds [10]. Furthermore, the effects of gibberellic acid and abscisic acid on germination and growth of okra seedlings under salinity stress have also been tested [11, 12]. Similarly, Durrant and Mash [9] stated that the application of 0.3M hydrochloric acid treatment increased the germination in the beet crop $[1,13,14]$. Moreover, the effect of different concentrations of gibberellic acid on the germination of two sunflower species was also reported $[12,15]$.

The present experiment aimed to investigate the effects of different treatments on dormancy breaking and germination inducing in okra, likewise, to measure the effects of the treatments on indicators such as dry and fresh weight of roots and shoots, root and shoot length, germination rate, percentage and vigor index.

\section{Materials and Methods}

This research was conducted in the laboratory of Samangan Institute of Higher Education, Samangan, Afghanistan in a completely randomized design with three replications in January 2020. The experiment was carried out under different treatments such as; gibberellic acid, hot water, cold and hydrochloric acid. First, the seeds were disinfected with a $10 \%$ sodium hypochlorite solution and then washed several times with distilled water. The treatments were applied as following; hot water (by immersing the seeds in hot water at $70{ }^{\circ} \mathrm{C}$ for 20 minutes), applying cold treatment $\left(3^{\circ} \mathrm{C}\right.$ for 7 days), placing the seeds in gibberellic acid (100, 500, 700 and $1000 \mathrm{ppm})$ for 12 hours, and seeds were placed in hydrochloric acid (75\%) for 5, 10 and 15 minutes. The germination test was performed in a completely randomized design with 3 replications (10 seeds per replication) in an incubator at $25^{\circ} \mathrm{C}$. For 20 days, germinated seeds were listed and on the 20 th day, the parameters such as; germination rate, germination percentage, vigor index, root and shoot length, fresh and dry weight of roots and stems were measured.

Equations 1, 2, and 3 were used to calculate the germination percentage, germination rate, and vigor index, respectively $[11,16]$.

$$
\begin{aligned}
& \text { Germination percentage }=\left(\frac{\text { number of germinated seeds }}{\text { total } \text { number of seeds }}\right) \times 100 \\
& \text { Germination rate }=\sum\left(\frac{\text { number of germinated seeds per day }}{\text { related day }}\right) \\
& \text { Seed vigor index }=\frac{\text { average seedling length in milimeter } \times \text { germination percentage }}{100 \%}
\end{aligned}
$$

The data were analyzed with SPSS v24. The significant differences were defined at $5 \%(p<0.05)$ using the Duncan Multiple Range Test, and the graphs were drawn by SPSS v24.

\section{Results}

\subsection{Root and Shoot Dry Weight:}

The effect of different treatments on root and stem dry weight was measured using the Duncan Multiple Range Test. However, among all treatments, only the 7-day cold treatment in root dry weight and the 10 minutes hydrochloric acid treatment in shoot dry weight showed significant differences with control, whereas the effects of all other treatments considered non-significant (Figure 1).

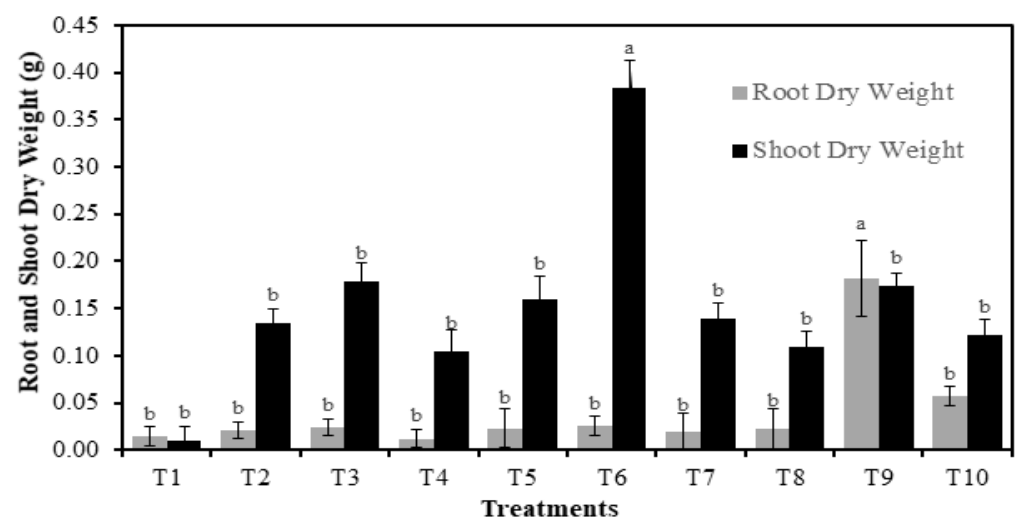

Figure-1.

The effect of treatments on the dry weight of roots and shoots.

T1: Control, T2: GA (100 ppm), T3: GA (500 ppm), T4: GA (700 ppm), T5: GA (100 ppm)T6: 7-days chilling, T7: Water $\left(70^{\circ} \mathrm{C}\right)$, T8: HCL (5mins), T9: HCL (10 mins), T10: HCL (15 mins). 


\subsection{Root and Shoot Fresh Weight}

Based on the result, there were highly significant differences in the root fresh weight for 10-minute hydrochloric and 7-day cold treatments compared to control, whilst in the case of shoot fresh root, that was only related to the 7-day cold treatments. On the other hand, the effects of gibberellic acid and cold treatments (particularly 500 ppm) were also showed a significant difference. As shown in Figure 2 the gibberellic acid at $500 \mathrm{ppm}$ concentration caused to increase the shoot fresh weight, however, with the high levels of the acid (700 to 1000 PPM) there was a decrease in the shoot weight, vice versa.

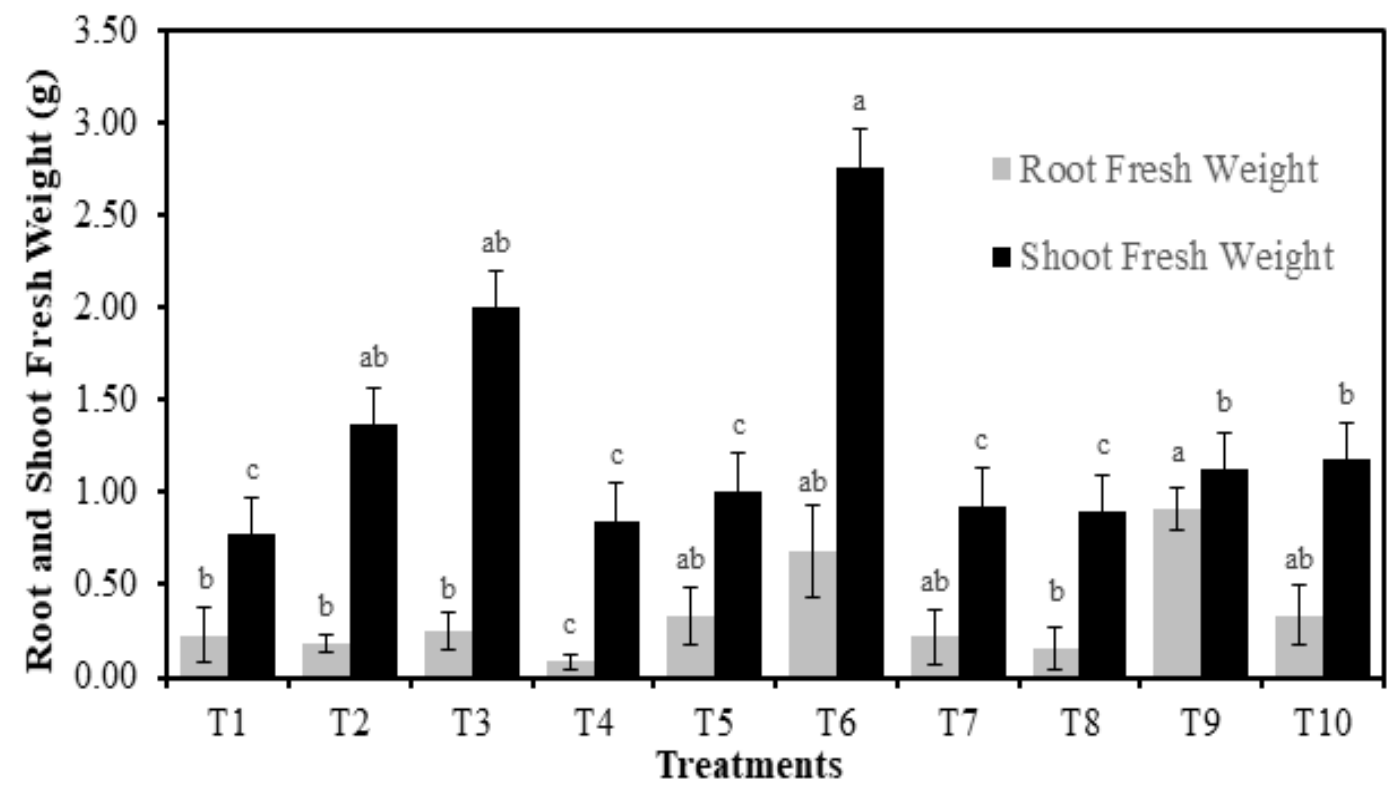

Figure-2.

The effect of treatments on fresh weight of roots and shoots.

T1: Control, T2: GA (100 ppm), T3: GA (500 ppm), T4: GA (700 ppm), T5: GA (100 ppm), T6: 7-days chilling, T7: Water (70 $\left.{ }^{\circ} \mathrm{C}\right)$, T8: HCL (5mins), T9: HCL (10mins), T10: HCL (15mins).

\subsection{Root and Shoot Length}

Regarding the root and shoot length, the 7-day cold treatment on the root length, and the treatments such as 7-days cold and 15-mins hydrochloric acid on shoot length had a significant difference compared to control (Figure 3).

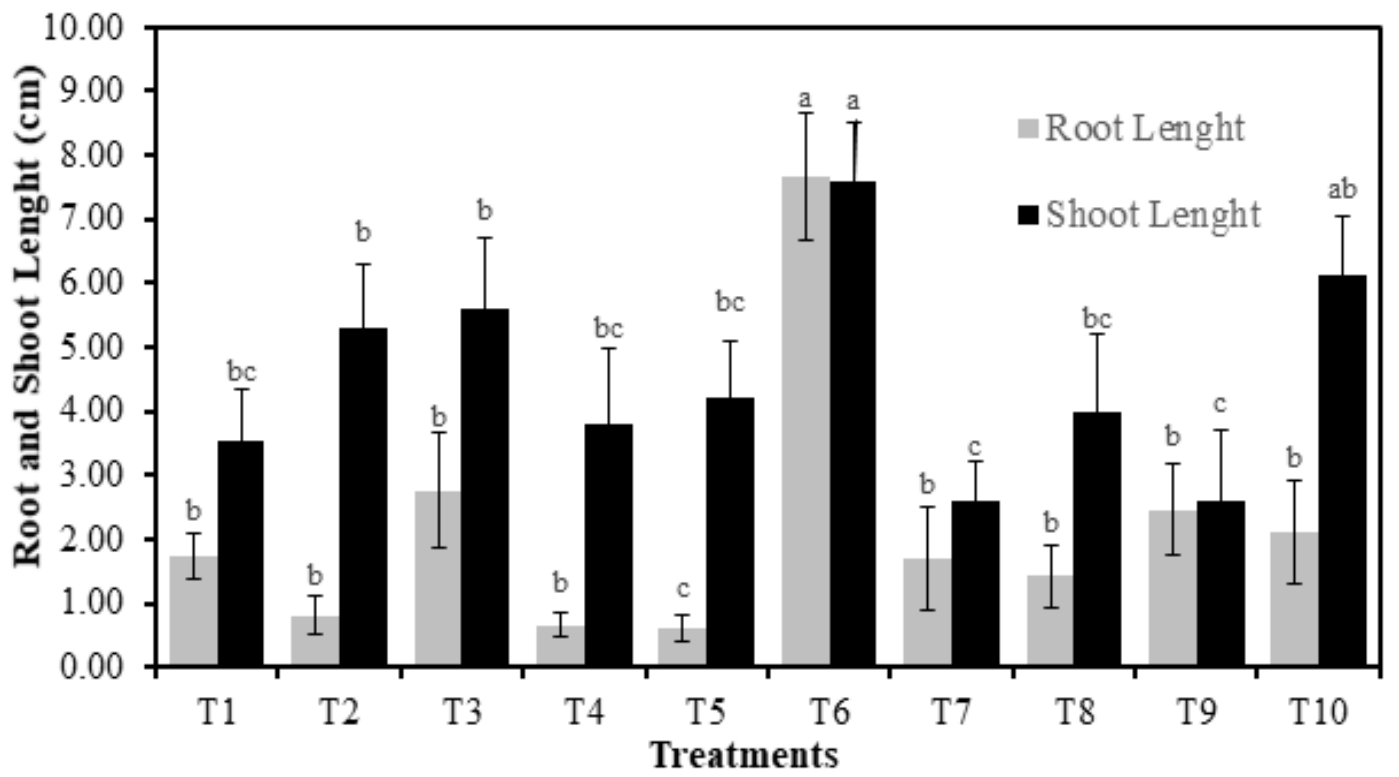

Figure-3.

Effect of treatments on root length.

T1: Control, T2: GA (100 ppm), T3: GA (500 ppm), T4: GA (700 ppm), T5: GA (100 ppm), T6: 7-days chilling, T7: Water (70 $\left.{ }^{\circ}\right)$,

T8: HCL (5mins), T9: HCL (10mins), T10: HCL (15mins).

\subsection{Germination Rate, Percentage, and Seed Vigor Index}

Based on the data, the effect of only 7-day cold treatment was considerably seen significant compared to control in all indices. While, the other treatments were all non-significant (Figures.4 and 5). 


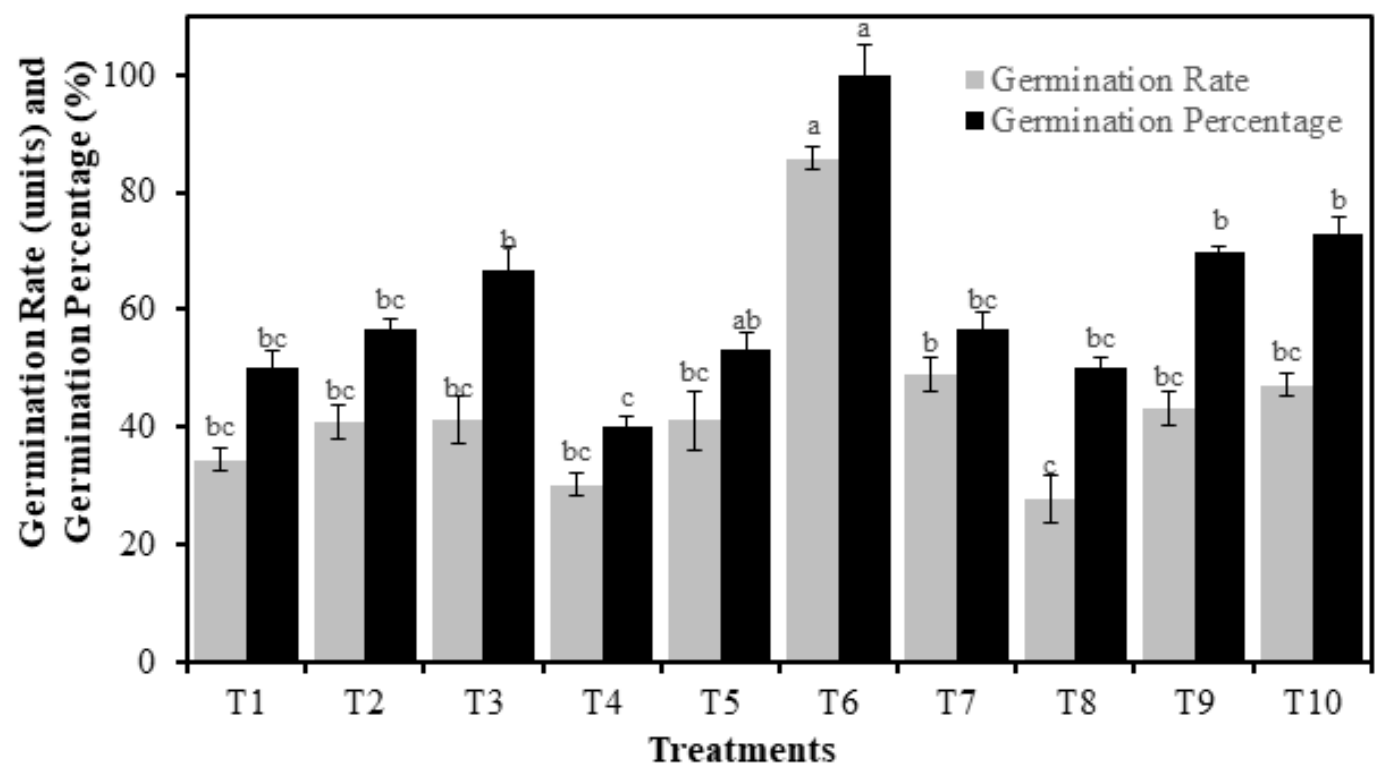

Figure-4.

The effect of treatments on germination rate and germination percentage.

T1: Control, T2: GA (100 ppm), T3: GA (500 ppm), T4: GA (700 ppm), T5: GA (100 ppm), T6: 7-days chilling, T7: Water $\left(70^{\circ} \mathrm{C}\right), \mathrm{T} 8$ : HCL (5mins), T9: HCL (10mins), T10: HCL (15mins).

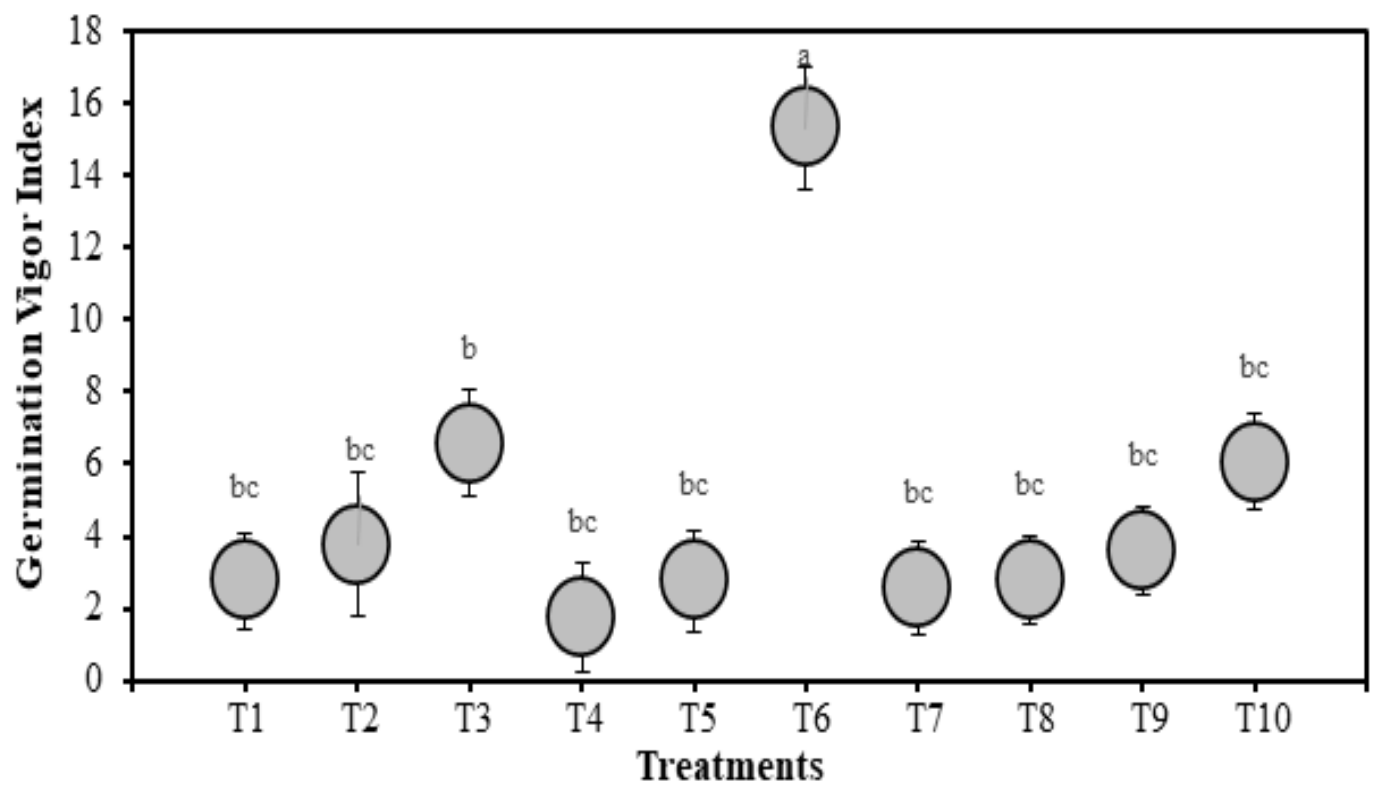

Figure-5.

The effect of treatments on the germination vigor.

T1: Control, T2: GA (100 ppm), T3: GA (500 ppm), T4: GA (700 ppm), T5: GA (100 ppm), T6: 7-days chilling, T7: Water (70 $\left.{ }^{\circ} \mathrm{C}\right)$, T8: HCL (5mins), T9: HCL (10mins), T10: HCL (15mins).

\section{Discussion}

The result of the present experiment is more consistent with the study of Durrant and Mash [9] which also reported the role of hydrochloric acid in increasing beet seeds germination [15, 17]. In this experiment, gibberellic acid at a concentration of $500 \mathrm{ppm}$ increased stem length and fresh stem weight. In a similar experiment, in the (Bauhinia rufescens) that the effect of the gibberellic acid concentrations at 500, 750, and $1000 \mathrm{ppm}$ on the indices such as stem diameter, stem fresh and dry weight, root fresh and dry weight, and the number of roots per seedling were examined, though except stem fresh weight index, the effects of all treatments on the indices were all considered non-significant [18, 19]; which is highly consistent with the result of this study. It might be because of the acid's property that causes the cell wall to soften and become more flexible, which leads to better cell growth in the process of cell elongation, and ultimately leads to better longitudinal growth of the stems [11].

In the root and shoot fresh and dry weight section, we have seen that the greatest effect was related to the 10 minutes hydrochloric acid and 7-days cold treatments, respectively. However, in terms of root and shoot length, germination rate and percentage, and vigor index, only 7-days cold treatment was seen to be effective rather than all other treatments. Hence, it seems that these treatments improve germination due to the dormancy breaking of seeds, therefore it ultimately leads to better stem and shoot growth. Based on the results, we found that cold is a better treatment to break the seed 
dormancy and improve germination. It seems that cold is responsible for activating the enzymes that stimulate the germination process [13] and thus might cause the growth of seedlings faster, affects the length of the stem, roots, and consequently in the stem and root weight.

\section{Conclusion}

Based on the findings of this experiment, it can be generally concluded that the cold had the greatest effect in all indices, while hydrochloric acid and gibberellic acid treatments were effective in indices such as root dry and shoot fresh weight, respectively. Therefore, considering the general results of the present study, a temperature of $3^{\circ} \mathrm{C}$ for 7 days can be suggested as the best way to break the dormancy of okra seeds and improve germination.

\section{References}

[1] A. Santner, L. I. A. Calderon-Villalobos, and M. Estelle, "Plant hormones are versatile chemical regulators of plant growth," Nature Chemical Biology, vol. 5, pp. 301-307, 2009.

[2] C. Job, A. Kersulec, L. Ravasio, S. Chareyre, R. Pepin, and D. Job, "The solubilization of the basic subunit of sugarbeet seed 11-S globulin during priming and early germination," Seed Science Research, vol. 7, pp. 225-244, 1997. Available at: https://doi.org/10.1017/s0960258500003585.

[3] C. Musara, J. Chitamba, and C. Nhuvira, "Evaluation of different seed dormancy breaking techniques on okra (Abelmoschus esculentus L.) seed germination," African Journal of Agricultural Research, vol. 10, pp. 1952-1956, 2015. Available at: https://doi.org/10.5897/ajar2014.9181.

[4] J. D. Bewley and M. Black, "Seeds, seeds germination, structure, and composition," ed Boston, MA: Springer, 1994, pp. 1-33.

[5] G. Mohammadi, E. M. Khah, S. J. Honarmand, A. Shirkhani, and G. Shabani, "Effects of seed hardness breaking techniques on okra (Abelmoschus esculentus L.) germination," International Journal of Agriculture and Crop Sciences, vol. 4, pp. 264-273, 2012.

[6] H. W. M. Hilhorst, "Definition and hypptheses of seed dormancy. K. Bradford, H. Nonogaki, Seed development, dormancy and germination. Annual plant reviews." vol. 27, ed Oxford: Blackwell Publishing, 2007, pp. 50-71.

[7] J. A. Opoku, J. N. Amissah, M. E. Essilfie, and J. C. Norman, "Effect of pre-sowing treatments on seed germination and seedling growth of silver butterfly tree (Bauhinia rufescens)," Current Agriculture Research Journal, vol. 6, pp. 344-354, 2018. Available at: https://doi.org/10.12944/carj.6.3.13.

[8] M. C. Saure, "Dormancy release in deciduous fruit trees," Horticultural Reviews, vol. 7, pp. 239-300, 1985. Available at: https://doi.org/10.1002/9781118060735.ch6.

[9] M. J. Durrant and S. J. Mash, "Sugar-beet seed steep treatments to improve germination under cold, wet conditions," Plant Growth Regulation, vol. 10, pp. 45-55, 1991. Available at: https://doi.org/10.1007/bf00035130.

[10] F. H. Peto, "Methods of loosening tight seed caps in mono-germ seed to improve germination," Journal of the American Society of Sugar Beet Technologists, vol. 13, pp. 281-286, 1964. Available at: https://doi.org/10.5274/jsbr.13.3.281.

[11] R. P. Adams and A. K. TeBeest, "The effects of different concentrations of gibberellic acid (GA3) on seed germination of Helianthus annuus and H. petiolaris," Phytologia, vol. 99, pp. 33-35, 2017.

[12] V. M. Jiménez, "Involvement of plant hormones and plant growth regulators on in vitro somatic embryogenesis," Plant Growth Regulation, vol. 47, pp. 91-110, 2005. Available at: https://doi.org/10.1007/s10725-005-3478-x.

[13] J. D. Bewley, "Seed germination and dormancy," The Plant Cell, vol. 9, pp. 1055-1066, 1997. Available at: https://doi.org/10.1105/tpc.9.7.1055.

[14] S. P. Groot, B. Kieliszewska-Rokicka, E. Vermeer, and C. M. Karssen, "Gibberellin-induced hydrolysis of endosperm cell walls in gibberellin-deficient tomato seeds prior to radicle protrusion," Planta, vol. 174, pp. 500-504, 1988.

[15] F. Yakoubi, F. Z. Babou, and M. Belkhodja, "Effects of gibberellic and abscisic acids on germination and seedling growth of Okra (Abelmoschus esculentus L.) under Salt Stress," Pertanika Journal of Tropical Agricultural Science, vol. 42, pp. 847860, 2019.

[16] K. A. I. Graeber, K. Nakabayashi, E. Miatton, G. E. R. H. A. R. D. Leubner-Metzger, and W. J. Soppe, "Molecular mechanisms of seed dormancy," Plant, Cell \& Environment, vol. 35, pp. 1769-1786, 2012. Available at: https://doi.org/10.1111/j.1365-3040.2012.02542.x.

[17] R. L. Geneve, "Seed dormancy in commercial vegetable and flower species," Seed Technology, pp. 236-250, 1998.

[18] M. Miransari and D. Smith, "Rhizobial lipo-chit oligosaccharides and gibberellins enhance barley (Hordeum vulgare L.) seed germination," Biotechnology, vol. 8, pp. 270-275, 2009. Available at: https://doi.org/10.3923/biotech.2009.270.275.

[19] L. O. Copeland and M. B. McDonald, "The chemistry of seeds, In principles of seed science and technology," ed Boston, MA: Springer, 2001, pp. 39-57. 